\title{
THE GRAPH DIAMETER OF A DISTRIBUTED SYSTEM WITH A GIVEN DOMINANT SET
}

\author{
A.M. Rappoport ${ }^{1}$, I.I. Kurochkin ${ }^{1, a}$ \\ ${ }^{1}$ Institute for Information Transmission Problems of Russian Academy of Sciences, \\ Bolshoy Karetny per. 19, build.1, Moscow, 127051, Russia \\ E-mail: ${ }^{a}$ qurochkin@gmail.com
}

In this work consider a distributed computing system in which the control functions are dispersed in several dominant nodes that are directly connected to all the others. This configuration reduces the vulnerability of the entire network, since the failure of a single control element immediately disrupts its operation. On the other hand, the large length of the maximum shortest chain (diameter) increases the data transfer time, which is bad for the functioning of the entire system. The connection of the maximum shortest chain of a distributed network graph with the size of a certain dominant set is investigated. The structure of a graph with a maximum diameter on the set of all graphs with a given dominant set is presented, a diametrical chain is constructed, and the value of the extreme diameter is estimated. Based on this construction, it is possible to generate various network graphs with a given dominant set and a diameter that takes certain values. A number of operations are proposed that change the edge set of the original graph. As a result, this method provides a way to construct graph structures with given metric characteristics.

Keywords: network graph, distributed computing network, distributed computing, dominant set, graph diameter, diametric chain construction 


\section{Introduction}

Distributed computing systems can be used to solve complex computational problems along with clusters and MPP systems [1]. It is necessary to take into account the features of distributed computing systems: the possibility of errors, the sudden shutdown of any computing node, the heterogeneity of computing nodes and significant restrictions on the data exchange [2]. There is "Bagof-Tasks" type of tasks for which calculations can be divided into many autonomous subtasks, and data exchange is not required [3]. But there are other types of tasks that need data exchange [4]. In this case, the design of a telecommunication network becomes one of the important goals in the functioning of a distributed system. This is achieved by using several types of nodes to perform certain functions: performing computations, store data, computing control, and data exchange. For example, structured distributed systems [5] may have several control nodes to ensure fault tolerance. The control nodes can be nodes with certain properties that belong to the dominant set of vertices of the distributed system graph [6,7]. One of the important characteristics of a telecommunications network graph is the length of the maximum shortest chain over all pairs of vertices or the diameter [8]. The diameter allows us to estimate the longest message transmission time in a network in which nodes function according to the disjunction logic [9]. Studies of the relationship between the minimum size of the dominant set for its small values and the diameter were devoted to [10,11].

In paper [11] a method was proposed for constructing a network with the maximum shortest chain (diametric) for the totality of all graphs with a given dominant set. Based on this approach, using a number of operations, it is possible to generate structures with a pre-known diameter value. This paper discusses one of these possibilities based on organization of interaction of a certain type between dominant nodes. It should be noted that the use of distributed computing systems, in which the control and coordinating functions are concentrated in several dominant elements directly connected to the rest, avoids excessive centralization and reduces the vulnerability of the entire network. In turn, the length of the maximum shortest chain (diameter) estimates the data transmission time if a message is transmitted when at least one is received. In this regard, it is advisable to investigate the question of the dependence of the diameter on the size of some dominant set [10].

\section{Configuration of graphs with maximum diameter}

Let's formulate the necessary concepts [ 9]. Let $G=G(V, E)$ - be a finite, connected undirected graph without multiple edges, $|V|=n,|E|=m$. By definition, diameter $\delta=\delta(G)=\operatorname{diam}(G)=\max \rho(x, y)$, where $\rho(x, y)$ - distance, i.e. the length of the shortest chain over all pairs of vertices $x, y \in V$. A dominant set $D$ is such a subset of vertices from $V$, that for any vertex $y \in V \backslash D$ there is an edge $(x, y) \in E, x \in D$. The minimum size of such a subset is called the dominance number $\gamma(G)$.

A method is given for constructing a graph with a maximum diameter on a set of graphs with a given dominant set of vertices [11]:

Let $D=\left\{x_{i}, i=\overline{1, s}\right\}$ is some dominating set of the required graph $G \cdot$ It has vertices from $D \subset V$ are not adjacent $\left(x_{i}, x_{j}\right) \notin E, i, j=\overline{1, s}$. Define in $V \backslash D$ disjoint "dominated" subsets of vertices $V_{i}=N\left(x_{i}\right)$ - the neighborhood of vertex $x_{i}$ and their corresponding subgraphs $G_{i}=G_{i}\left(V_{i}, E_{i}\right)$ , by construction $V_{i} \cap V_{j}=\varnothing, E_{i} \cap E_{j}=\varnothing, i, j=\overline{1, s}$.

We require the fulfillment of additional conditions on the structure of these subgraphs. And let's designate them (A) together with the already formulated conditions.

In these subgraphs, the vertices are divided into 2 disjoint subsets: $V_{i}=V_{i}^{1} \cup V_{i}^{2}$, and vertices 
from different subsets are not adjacent. In subgraphs $G_{i}^{1}=G_{i}^{1}\left(V_{i}^{1}, E_{i}^{1}\right), G_{i}^{2}=G_{i}^{2}\left(V_{i}^{2}, E_{i}^{2}\right)$ only interior edges are allowed, and at least one vertex from $V_{i}^{2}$ is adjacent to a vertex from $V_{i+1}^{1}$. The main result can be formulated as Theorem 1 .

\section{Theorem 1}

If graph $G=G(V, E)$ with dominant set $D=\left\{x_{i}, i=\overline{1, s}\right\}$ satisfies the conditions (A), then the length of the maximal shortest path (diametrical) in it is equal to $3 s-1$. This chain is: $\left\{y_{1}^{1}, x_{1}, y_{1}^{2}\right.$, $\left.y_{2}^{1}, x_{2}, y_{2}^{2}, \ldots, y_{s-1}^{1}, x_{s-1}, y_{s}^{2}, x_{s}, y_{s}^{1}\right\}$, where $y_{i}^{1} \in V_{i}^{1}, \quad y_{i}^{2} \in V_{i}^{2}$.

In addition, on the set of all graphs with such a dominant set $D$ the diameter $\delta \leq 3 s-1$. A similar result on the estimation of the diameter was previously known [10].

\section{Building a network with a certain diameter}

As one of the possible ways to obtain a configuration with a given value of the diameter, one can use the introduction on the set of vertices of the dominant set $D$ of the edges of the complete subgraph $K_{p}$ with $p$ vertices $2 \leq p \leq s$. This will make it possible to reduce the length of the diametrical chain in the new graph $G_{p}$.

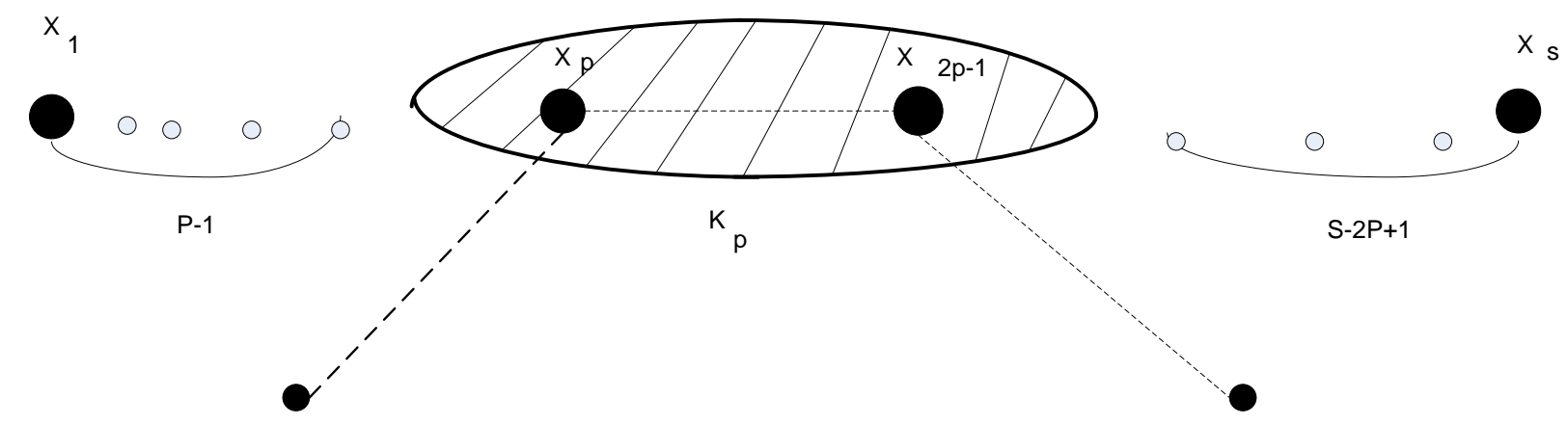

Figure 1. Dominant set

Figure 1 schematically shows the dominant set $D,|D|=$ s with edges from the diametrical chain between the vertices $x_{1}$ and $x_{s}$, incident to the vertices $x_{p}$ and $x_{2 p-1}$, including the edge between them from the complete subgraph $K_{p}$. Then this chain, following Theorem 1, is obtained by removing $3 p-1$ an edge from a chain of length $\delta=3 s-1$ and adding 3 edges shown by the dotted line. Therefore, its length is $\delta\left(G_{p}\right)=3 s-1-(3 p-1)+3=3(s-p+1)$. This implies Theorem 2:

\section{Theorem 2}

In graph $G_{p}=G\left(K_{p}\right)$ with dominant set $D,|D|=$ s on $\mathrm{p}$ vertices of which a complete subgraph $K_{p}$ is constructed with diameter $\delta\left(G_{p}\right)=3(s-p+1)$, and the diametrical chain between $x_{1}$ and $x_{s}$ consists of 3 fragments, and the middle fragment contains an edge $\left(x_{p}, x_{2 p-1}\right)$ from $K_{p}$.

For illustration, consider the case when $p=2$ and $s=4$, that is $\left(x_{p}, x_{2 p-1}\right)=\left(x_{2}, x_{3}\right)-$ edge in $G_{p}$. 


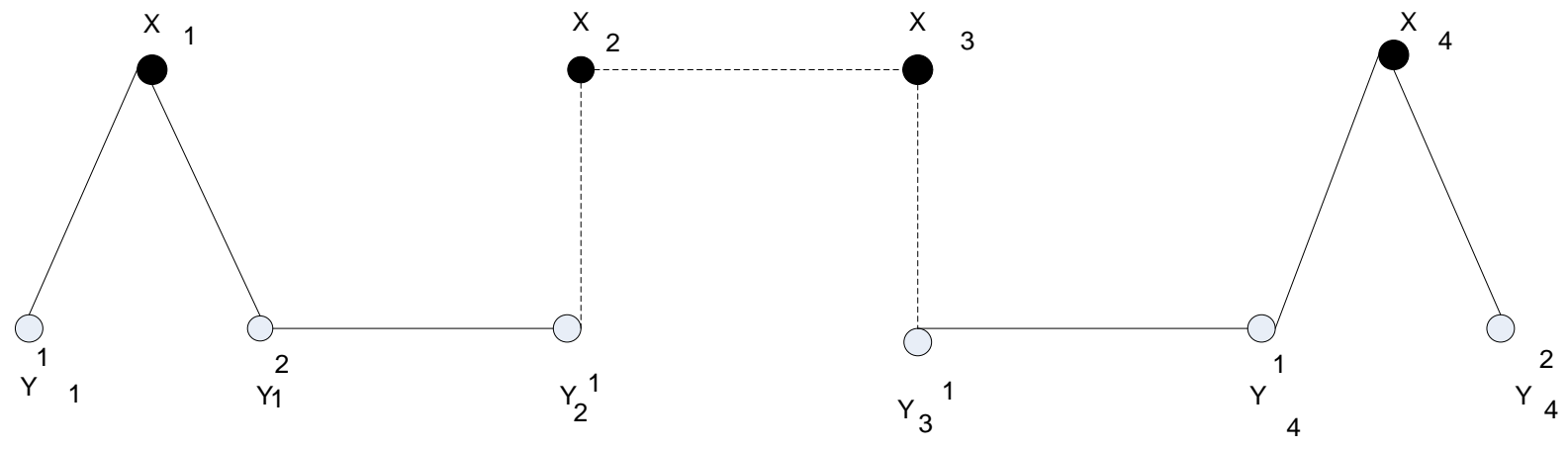

Figure 2. Graph with $\mathrm{p}=2, \mathrm{~s}=4$

In an extreme graph, when there are no edges between vertices from $D \rho\left(x_{1}, x_{4}\right)=11$, and in the constructed graph $G\left(K_{2}\right)$ the length of the maximal chain (diametrical) $\rho\left(x_{1}, x_{4}\right)=9$.

\section{Conclusion}

A method was proposed for constructing a graph with a maximum diameter on a set of graphs with a given size of the dominant set. The use of this method of constructing a graph of a telecommunication network allows avoiding both excessive centralization. It becomes possible to assess the time of data transmission in the network, with nodes operating on the basis of disjunctive logic. The structure of extreme graphs and estimates of parameters are presented: the number of edges and vertices, the type of the diametrical chain and the value of the maximum diameter. The results obtained were applied to graphs with two dominant vertices. The proposed approach can be used to construct graphs of telecommunication networks of structured distributed systems with a known dominant set and a certain value of the diameter.

\section{Acknowledgement}

The reported study was funded by RFBR according to the research project No. 19-07-00834.

\section{References}

[1] Foster I., Kesselman C. (ed.). The Grid 2: Blueprint for a new computing infrastructure. Elsevier, 2003.

[2] Li S. et al. A fundamental tradeoff between computation and communication in distributed computing //IEEE Transactions on Information Theory, Vol. 64, No.1, 2017. - pp. 109-128.

[3] Anderson, D.P.: BOINC: A Platform for Volunteer Computing. J. Grid Comput. (2020). DOI: 10.1007/s10723-019-09497-9.

[4] Sukhoroslov O. Supporting Efficient Execution of Workflows on Everest Platform // Communications in Computer and Information Science, Springer, Cham, Vol.1129, 2019. - pp. 713-724.

[5] Al Mojamed M., Kolberg M. Structured Peer-to-Peer overlay deployment on MANET: A survey //Computer Networks, Vol. 96, 2016. - pp. 29-47.

[6] Haynes T. W., Hedetniemi S., Slater P. Fundamentals of domination in graphs. - CRC press, 1998. 
[7] Haynes T. W. Domination in Graphs: Volume 2: Advanced Topics. - Routledge, 2017.

[8] West D. B. et al. Introduction to graph theory. - Upper Saddle River, NJ : Prentice hall, Vol.2, 1996.

[9] Goles E., Noual M. Disjunctive networks and update schedules //Advances in Applied Mathematics, Vol. 48, No. 5, 2012. - pp 646-662.

[10] Afanasiev A.P., Rappoport A.M. Construction and estimation of dominating sets in communication structures // DOKLADY MATHEMATICS, Pleiades Publishing, Ltd. 2006. Vol. 408, No.6, pp.746-749.

[11] Rappoport A.M., Gnedenko L.S., Kurochkin I.I. Construction of a communication network with a given dominant set and maximum shortest chain // Journal of high performance computing systems and technologies, Vol. 4, No.2, 2020. ISSN 2619-0818 (in Russian) 